\title{
IONIZATION ENERGIES OF RE IONS IN WIDE BANDGAP SULPHIDES *
}

\author{
K. ŚWIAteK aNd M. GodlewsKi \\ Institute of Physics, Polish Academy of Sciences, Al. Lotników 32/46, Warszawa, Poland
}

(Received August 8, 1990)

\begin{abstract}
An analysis of rare earth (RE) energy level positions in wide bandgap sulphides is presented. It is shown that the Jörgensen's refined spin-pairing energy theory (RESPET) predicts correctly the photo-ionization (PI) energy of $\mathrm{Sm}$ in $\mathrm{ZnS}$.
\end{abstract}

\section{PACS numbers: 71.55.Gs, 78.55.Et}

The crucial point in selecting appropriate host materials and dopants for electroluminescent devices is the knowledge of the ionization energies of impurity ions from which an efficient emission may be obtained. $\mathrm{RE}$ ions represent a relatively simple system of dopants in wide bandgap materials. Due to the screening of the $4 f^{q}$ shell by the external $5 s$ and $5 p$ shells, all electronic and magnetic properties are either identical or close to those of free ions, and depend rather weakly on lattice ionicity, lattice parameters, and structure. It was carefully tested that the energies of intra-ion and ionization transitions of a given RE ion are similar or nearly identical in crystals with the same ligand and independent of lattice "details" $[1,2]$.

Recently, we have shown that for $\mathrm{Eu}, \mathrm{Tm}$, and $\mathrm{Yb}$ the $\mathrm{RE}^{2+/ 3+}$ energy level is located in the forbidden gap of $\mathrm{ZnS}$ [3]. The energies of appropriate PI (charge transfer) transitions calculated basing on the concepts of Jorgensen's RESPET model $[1,4]$ coincide very well with the experimental values [5-7]. In this model, after correction for multiplet splitting $\left(\Delta E_{\mathrm{MC}}\right)$ of the initial and final states, the ionization energy depends linearly on " $q$ ", i.e. on the number of electrons on the $4 f$ shell of the RE ion. $\Delta E_{\mathrm{MC}}$ can be easily calculated since the appropriate Racah and Lande parameters were given by Carnall et al. [8]. The linear term $(E-A)$ in $q$ approximates three important contributions to RE ionization energy:

- the regular $Z$ - and $q$-dependent increase due to the contraction of the RE ionic radius,

\footnotetext{
*This work was supported by CPBP 01.12.
} 
- the regular $Z$ - and $q$-dependent increase in the effective charge felt by $4 f$ electrons,

- the regular $q$-dependent variation in $4 f$-shell intra-electronic repulsion.

The $W$ term in eq.(1) for the $3+\Rightarrow 2+$ ionization enthalpy $\left(\Delta H_{\text {II-III }}\right)$ describes the ionization energy of the RE ion, the negative electrostatic potential energy at the ligand, polarization energy of the RE-ligand system and the lattice relaxation energy.

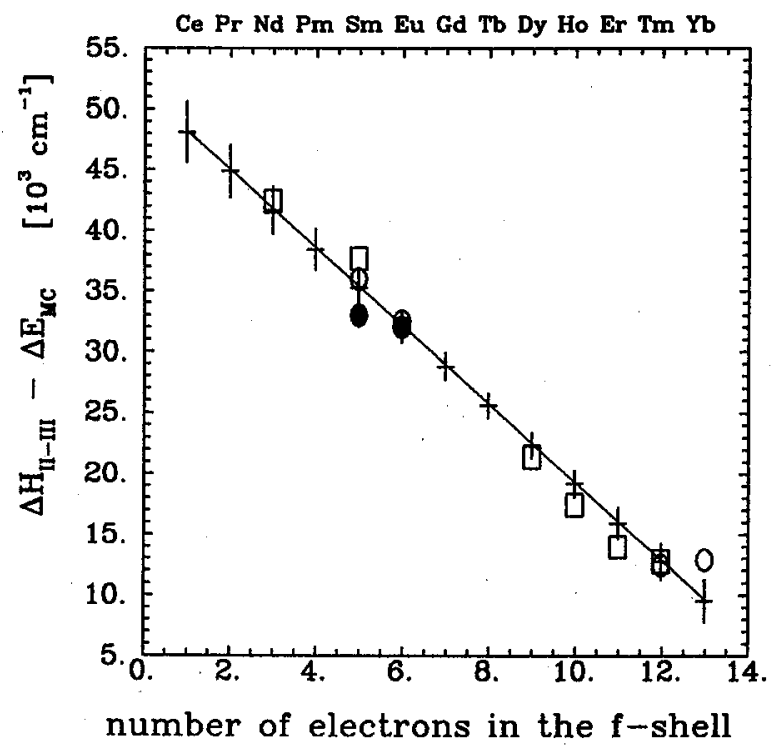

Fig. 1. Ionization enthalpy minus multiplet correction $\left(\Delta H_{\mathrm{II}-\mathrm{III}}-\Delta E_{\mathrm{MC}}\right)$ for $3+\Rightarrow 2+$ $\mathrm{RE}$ transitions in $\mathrm{ZnS}, \mathrm{SrS}$, and $\mathrm{CaGa}_{2} \mathrm{~S}_{4}$. Experimental data: (o) for $\mathrm{ZnS}$ [5-7, 9], (•) for SrS [10], and (ㅁ) for $\mathrm{CaGa}_{2} \mathrm{~S}_{4}$ [11]. Theoretical data: (-) fit to the edge of photoluminescence excitation bands.

$$
\Delta H_{\mathrm{II}-\mathrm{III}}-\Delta E_{\mathrm{MC}}=W+q(E-A)
$$

The basic assumption of the RESPET approach is that $\Delta E_{\mathrm{MC}}, W$, and ( $E-$ A) are similar for all RE systems with the same ligand or for systems with different ligands but of similar electronegativities (e.g. sulphur and iodine). Once we know the ionization energies of some RE ions for a given ligand the ionization energies of other RE ions can be calculated from eq.(1) by first calculating $\Delta E_{\mathrm{MC}}$ for each $\mathrm{RE}$ and then by taking $W$ and $(E-A)$ as parameters determined from the fit to the experimental results.

From the photo-EPR experiments we know the positions of $\mathrm{Eu}^{2+/ 3+}$ and $\mathrm{Yb}^{2+/ 3+}$ energy levels in $\mathrm{ZnS}[5,7]$. In our previous work [3] we have shown that the maximum around $26000 \mathrm{~cm}^{-1}$ in the excitation spectrum of the $\mathrm{Tm}^{3+}{ }^{1} \mathrm{G}_{4} \Rightarrow^{3} \mathrm{II}_{6}$ 


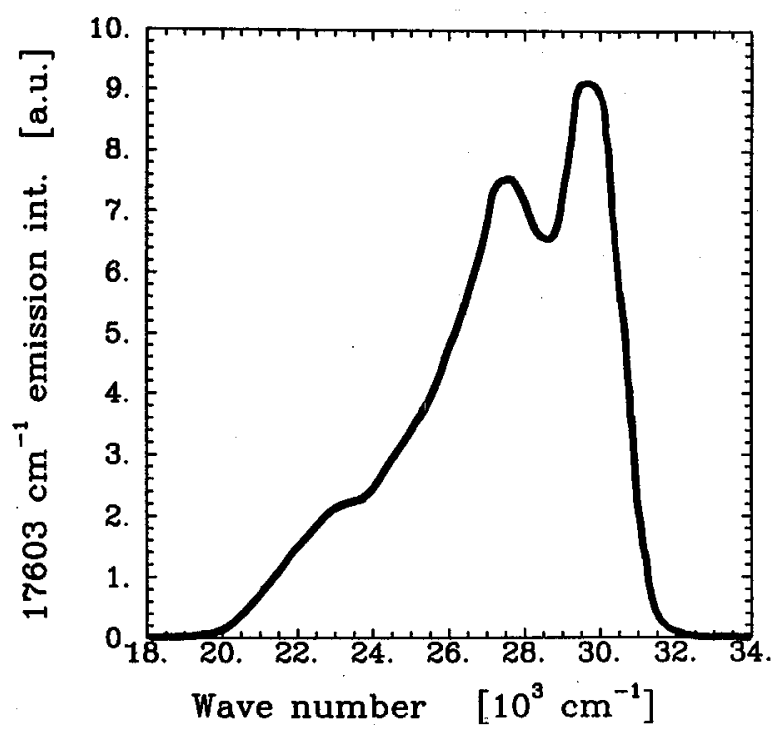

Fig. 2. Excitation spectrum of the main $\mathrm{Sm}^{3+}{ }^{4} \mathrm{G}_{5 / 2} \Rightarrow{ }^{6} \mathrm{H}_{9 / 2}$ emission in $\mathrm{ZnS}$ measured at $4.2 \mathrm{~K}$.

emission in $\mathrm{ZnS}$, observed by Zimmermann and Boyn [6], should be attributed to $\mathrm{Tm}^{3+}+h \nu \Rightarrow \mathrm{Tm}^{2+}+h_{\mathrm{vb}}$ PI transition. To approximate the $\Delta H_{\mathrm{II}-\mathrm{III}}-\Delta E_{\mathrm{MC}}$ value for the $\mathrm{Sm}$ ion in $\mathrm{ZnS}$ lattice we performed the fit with RESPET, firstly, to the data for $\mathrm{Eu}, \mathrm{Tm}$, and $\mathrm{Yb}$ ions in $\mathrm{ZnS}$ and, secondly, to the set of all available data for $\mathrm{RE}$ ions in $\mathrm{ZnS}$, SrS, and $\mathrm{CaGa}_{2} \mathrm{~S}_{4}$ lattices (Fig. 1). We have obtained the following values of $\Delta H_{\mathrm{II}-\mathrm{III}}: 22054 \pm 17847 \mathrm{~cm}^{-1}$ and $22025 \pm 1466 \mathrm{~cm}^{-1}$ for $\mathrm{Sm}$ ion in the first and second case, respectively. The given errors of the values correspond to appropriate variances in the least square analysis. The estimated energy of $\mathrm{Sm}$ ionization transition in sulphides agrees quite well with the position of the excitation band of $\mathrm{Sm}^{3+{ }^{4}} \mathrm{G}_{5 / 2} \Rightarrow{ }^{6} \mathrm{H}_{9 / 2}$ emission in the $\mathrm{ZnS}$ lattice (Fig. 2) [9]. Thus, we conclude that similarly as for $\mathrm{Tm}$ in $\mathrm{ZnS}$ [6], the efficient photoluminescence excitation mechanism is due to RE ionization and subsequent recombination via the excited state.

\section{References}

[1] C.K. Jörgensen, Mol. Phys. 5, 3 (1962).

[2] C.K. Jörgensen, in Structure and Bonding, Vol.22, eds. J.D. Dunitz et al., Springer Verlag, Berlin, IIeidelberg, New York 1975, p. 49.

[3] K. Świątek, M. Godlewski, Acta Phys. Pol. A77, 95 (1990).

[4] J.L. Ryan, C.K. Jörgensen, Mol. Phys. 7, 17 (1963).

[5] M. Godlewski, D. Hommel, Phys. Status Solidi A 95, 261 (1986). 
[6] H. Zimmermann, R. Boyn, Phys. Status Solidi B 135, 379 (1986).

[7] H. Przybylińska, K. Świątek, A. Stąpor, A. Suchocki, M. Godlewski, Phys. Rev. B 40, 1748 (1989).

[8] W.T. Carnall, P.R. Fields, K. Rajnak, J. Chem. Phys. 49, 4424 (1968).

[9] K. Świątek, M. Godlewski, D. Hommel, Phys. Rev. B 41 (March 1991).

[10] R.S. Title, Phys. Rev. Lett. 3,273 (1959).

[11] A. Garcia, F. Guillen, C. Fouassier, J. Lumin. 33, 15 (1985). 